\title{
Optical Properties of Chalcogenides
}

\author{
Sikander Azam* \\ Department of Physics, Pakistan \\ *Corresponding author: Sikander Azam, Department of Physics, Riphah International University, Lahore, Pakistan
}

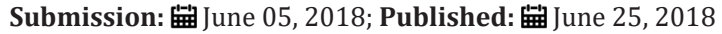

Abstract

Advancement in optical telecommunications and optical computing devices has increased the demand for materials with greater optical coefficients. For that reason, several materials with suitable optical properties have been designed, some of which are based on the family of quaternary chalcogenide compounds. Their flexibility in varying composition as well as structure allows tuning their optical properties.

\section{Introduction}

From the very beginning of evolution, humans worked on the physical properties of materials in order to ensure that they meet their needs and this remains a key field of interest for scientists today. Thus far, scientists have succeeded in obtaining efficient materials for different technological applications and are still working on further improvement in this field.

Pn-junction and transistor brought a revolution in the field of condensed matter physics. The most valuable work in science, especially in the field of condensed matter, pertains to the semiconductor chips (integrated circuits). Today, many electronic devices are of very small size, due to the use of semiconductors in the electronic circuits, which also make these devices more efficient and reliable. Technological and industrial development relies on the advances made in the field of semiconductors, magnetic and metallic materials, which are the basic building blocks of electronic devices. Promising devices are based on the chalcogenide compounds, owing to their advantageous properties.

Chalcogenide materials, i.e., those containing the chalcogen elements (S,Se, or Te), are widely investigated for their applications in technological uses, for instance, thermoelectric, optoelectronic, photoelectric, phosphors, and solid-state electrolytes for lithium secondary batteries, among others. Chalcogenide compounds have a very diverse and interesting structural chemistry and possess useful physical and chemical properties that may be applicable to modern technologies [1]. They have received much attention during the past decade, largely because of their optoelectronic and thermoelectric properties.

These materials have wide variations in their energy gaps (Eg), ranging from ultraviolet (UV) to infrared (IR). Such properties are useful in the development of light emitting diodes (LED), laser diodes, optoelectronic devices and optical detectors. Most such compounds, especially metal sulfides, selenides and tellurides, belong to a large class of compounds known as metal chalcogenides. Sulfur, being able to catenate and bind to several metal centers, has great importance in chemistry of metal chalcogenides, and similar nature has been determined for Se and Te [1,2].

Metalsulfur compounds have been widely studied in chemistry due to their important properties. Significance of these compounds has been enhanced owing to their unique and diverse properties and their vital role in hydro-desulfurization, bioinorganic chemistry and other catalytic methods. Complexes of molecular transition metal with terminal/bridging sulfide ligands have been extensively studied for their catalytic activities [3,4]. Using the solid-state approach or in solution, soluble metal fluorides and sulfides have been synthesized and their properties demonstrated $[5,6]$. Some compounds, including selenium and tellurium, have structure types that are presently unknown in sulfur chemistry. Tellurium, due to its large size, diffused orbitals and greater metallic nature relative to other chalcogen elements, is characterized by very different non-classical chemistry.

Most of low-dimensional materials have been greatly modified by covalent bond nature of metal chalcogenides. The valence electrons of $4 \mathrm{~d}$ - and $5 \mathrm{~d}$-elements make them highly suitable for forming M-M (metal-metal) bonds, which are restricted to directly coupled atoms, and clusters in particular. Such types of clusters are found in discrete molecular units joined by bridging ligands [7]. Chalcogenide clusters are presently the best known inorganic or high valence clusters and are typical for 4 dand $5 \mathrm{~d}$-metals of groups V-VII $[8,9]$. 


\section{Conclusion}

The frequency dependent dielectric function was calculated to study optical properties. The calculated results showed inter/intraband absorption in the low frequency region within the band gap..

\section{References}

1. Whittingham (1978) Solid State Chem 12: 60.

2. Fritz IJ, Isaacs TJ, Gottlieb M, Morosin B (1978) Solid State Commun 27: 535.

3. Rabenau, Angew A (1985) Chem 24: 1026.

4. DuBois MR (1989) Catalytic applications of transition-metal complexes with sulfide ligands. Chem Rev 89(1): 1-9.
5. Schultz LD, Koehler WH (1987) Synthesis and characterization of sodium polytellurides in liquid ammonia solution. Inorg Chem 26(12): 1989-1993.

6. Kadel K, Kumari L, Li WZ, Huang JY, Provencio PP (2011) Nanoscale Res Lett 6: 57.

7. Simon, Angew A (1981) Chem 20: 1.

8. (2007) Chem Inform 38(52).

9. Klchambare P, Sharon M, Seki Y, Hagino T, Nagata S (1997) Preparation and fundamental physical properties of the quaternary chalcogenides $\mathrm{SnBi}_{4-\mathrm{x}} \mathrm{Te}_{3+\mathrm{y}} \mathrm{Se}_{4-\mathrm{z}}$ and $\mathrm{PbBi}_{4-\mathrm{x}} \mathrm{Te}_{3-\mathrm{y}} \mathrm{Se}_{4-\mathrm{z}}$. Solid State lonics 101-103(1): 125-129.
Creative Commons Attribution 4.0 International License

For possible submissions Click Here

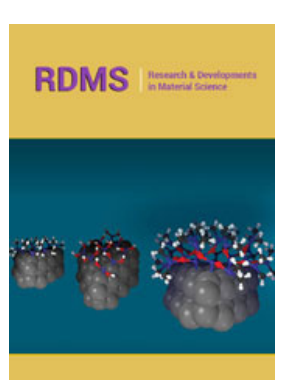

Research \& Development in Material Science Benefits of Publishing with us

- High-level peer review and editorial services

- Freely accessible online immediately upon publication

- Authors retain the copyright to their work

- Licensing it under a Creative Commons license

- Visibility through different online platforms 